\title{
GRANDE-BRETAGNE
}

\section{J.-II. POI'TER}

Le chirurgien-major J.-H. Porter, mort le 9 janvier dernier, étail entré au service médical de l'armée anglaise en 1853: Il fit la campagne de Crimée; plus tard il servit dans les nombrevix engagements amenés par l'insurrection des Indes, y compris le siége et la prise de Lncknow et l'assaut de Kaiser-Bagh. Pendant la guerre franco-allemande, il prit une part volontaire au service de l'ambulance neutre instituée par la Sociélé de la Ciroix rouge anglaise. En 187\%, il fut appelé comme professeur de chirurgie militaire dans l'école de Nelley; il s'y distingua comme habile opérateur et conserva ce poste jusqu'en 1878. Il fut alors appelé aux Indes, au service aclif de l'armée qui opérait dans l'Afghanistan, et c'est là qu'il est mort. Son travail le plus remarquable a été l'organisation du service des ambulances, de concert avec l'inlendance générale (Quarter muster General's Department), pour le retour des troupes, qui devait être elfectué par détachements successifs.

Après la conclusion de la paix avec l'émir de Caboul, le $\mathrm{D}^{\mathrm{r}}$ Porter fut signalé par -ses supérieur's militaires, pour l'énergie et l'habileté qu'il avait déployées dans ses délicates fonctions.

Lor's du massacre de l'ambassade anglaise à Caboul, il reçut subitement l'ordre d'aller rejoindre le quartier-général du général Roberts. C'est là que commenga pour lui la nouvelle tâche d'organiser les hòpitaux de campagne et les moyens de transport dans la direction de Caboul. Dans une attaque qui eut lieu pendant cette marche en avant, le $D^{r}$ Fownsend, médecin en chef de la division, ayant été grièvement blessé à la figure, le $\mathrm{D}^{\mathrm{r}}$ Porter dut prendre sa place et remplir les fonctions de médecin en chef de l'armée anglaise devant Caboul. Toutes les responsabilités premières dans l'ordre médical pesèrent dès lors sur lui, pendant cette périlleuse campagne.

Less rapports parvenus en Angleterre témoignent unanimement 
que les ordres donnés et les mesures prises par le $\mathrm{D}^{\mathrm{r}}$ Porter furent l'eçus avec une respectueuse confiance. Il ne pouvait en ètre autrement de la part de tous ceux qui connaissaient sa grande expérience pratique, son bon sens, son tact et sa parfaite discrétion, comme aussi son dévouement à tonte épreuve pour tous les malheureux conflés à ses soins.

On peut done bien affirmer que, dans la personne du chirurgien-major Porter, l'armée de Caboul et le Département militaire ont perdu un de leurs officiers les plus distingués.

Il s'était d'ailleurs fait comnâtre par diverses jublications chirurgicales généralement estimées, et spécialement par son remarquable Manuel des médecins militaires en campagne. Nos lecteurs n'ont pas oublié que cet ouvrage fut honoré du second prix institué par l'impératrice d'Allemagne pour le meilleur manuel de chirurgie militaire.

Nommé en 1875 associé honoraire de l'Ordre de St-Jean en Angleterre, le $\mathrm{D}^{r}$ Porter y était devenu un nembre très-apprécié du Comité central pour l'organisation des ambulances.

Après les détails précédents, extraits d'un journal anglais, il nous sera permis d'ajouter que le Comité international de la Croix rouge perd, dans l'homme dont il déplore la mort subite, un sympathique défenseur, et nous-même personnellement un digne confrère; nous aimions à le retronver dans les diverses conférences internationales convoquées pour les questions relatives au soin des blessés, el à échanger avec lui des publications médicales.

Le Dr Porter est mort an champ d'honneur en remplissant le plus noble des devoirs; ce que nous désirons pour nous, c'esi de suivre son exemple!

Docteur Lis Apra.

\section{GRECE}

APPEL EN Faveur des RÉfugiés CIIRÉTIENS EN GRÉce

Tous les Grecs, ainsi que les étrangers qui suivent les événements en Orient, connaissent les circonstances sous l'empire desquelles plusieurs milliers de chrétiens des provinces limitrophes se 